\title{
Partial neutral functional integro-differential equations of fractional order with delay
}

\author{
Saïd Abbas ${ }^{1}$, Mouffak Benchohra² and Alberto Cabada ${ }^{3 *}$
}

\section{"Correspondence:}

alberto.cabada@usc.es

${ }^{3}$ Departamento de Análise

Matemática, Facultade de

Matemáticas, Universidade de

Santiago de Compostela, Santiago

de Compostela, Spain

Full list of author information is

available at the end of the article

\begin{abstract}
In this paper we obtain sufficient conditions for the existence of solutions of some classes of partial neutral integro-differential equations of fractional order by using suitable fixed point theorems.

MSC: $26 \mathrm{~A} 33$

Keywords: integro-differential equation; left-sided mixed Riemann-Liouville integral of fractional order; Caputo fractional-order derivative; finite delay; infinite delay; solution; fixed point
\end{abstract}

\section{Introduction}

Fractional differential and integral equations have recently been applied in various areas of engineering, science, finance, applied mathematics, bio-engineering and others. There has been a significant development in ordinary and partial fractional differential equations in recent years; see the monographs of Abbas et al. [1], Baleanu et al. [2], Kilbas et al. [3], Lakshmikantham et al. [4], Podlubny [5], and the references therein.

In [6], Czlapinski proved some results for the following system of the Darboux problem for the second-order partial functional differential equations of the form

$$
\begin{aligned}
& D_{x y}^{2} Z(x, y)=f\left(x, y, Z_{(x, y)},\left(D_{x} Z\right)_{(x, y)},\left(D_{y} Z\right)_{(x, y)}\right) ; \quad(x, y) \in \mathbb{R}^{2}, \\
& Z(x, y)=\Phi(x, y) ; \quad \text { if }(x, y) \in E^{0}:=((-\infty, a] \times(-\infty, b]) \backslash((0, a] \times(0, b]),
\end{aligned}
$$

where $a, b>0, f: \mathbb{R} \times \mathcal{B} \times \mathcal{B} \times \mathcal{B} \rightarrow \mathbb{R}, \Phi: E^{0} \rightarrow \mathbb{R}, D_{x}:=\frac{\partial}{\partial x}, D_{y}:=\frac{\partial}{\partial y}, D_{x y}^{2}:=\frac{\partial^{2}}{\partial x \partial y}$, and $\mathcal{B}$ is a vector space of real-valued functions defined in $(-\infty, 0] \times(-\infty, 0]$, equipped with a seminorm and satisfying some suitable axioms, which was introduced by Hale and Kato [7]; see also [8-10] with rich bibliography concerning functional differential equations with infinite delay. Recently, Abbas et al. studied some existence results for the Darboux problem for several classes of fractional-order partial differential equations with finite delay $[11,12]$ and others with infinite delay $[13,14]$.

Motivated by the above papers, in this article we deal with the existence of solutions for two systems of neutral integro-differential equations of fractional order with delay. First, we consider the system of fractional-order neutral integro-differential equations with finite delay of the form

$$
{ }^{c} D_{\theta}^{r}\left[u(x, y)-g\left(x, y, u_{(x, y)}\right)\right]=f\left(x, y, I_{\theta}^{r} u(x, y), u_{(x, y)}\right) ; \quad(x, y) \in J,
$$

๑) 2012 Abbas et al; i licensee Springer. This is an Open Access article distributed under the terms of the Creative Commons Attribution License (http://creativecommons.org/licenses/by/2.0), which permits unrestricted use, distribution, and reproduction in any medium, provided the original work is properly cited. 


$$
\begin{aligned}
& u(x, y)=\Phi(x, y) ; \quad \text { if }(x, y) \in \tilde{J}:=([-\alpha, a] \times[-\beta, b]) \backslash((0, a] \times(0, b]), \\
& \begin{cases}u(x, 0)=\varphi(x) ; & x \in[0, a], \\
u(0, y)=\psi(y) ; & y \in[0, b], \\
\varphi(0)=\psi(0),\end{cases}
\end{aligned}
$$

where $J:=[0, a] \times[0, b] ; a, b, \alpha, \beta>0, \theta=(0,0), r=\left(r_{1}, r_{2}\right), r_{1}, r_{2} \in(0, \infty), I_{\theta}^{r}$ is the leftsided mixed Riemann-Liouville integral of order $r$ (see Section 2 for definition), ${ }^{c} D_{\theta}^{r}$ is the fractional Caputo derivative of order $r, f: J \times \mathbb{R}^{n} \times \mathcal{C} \rightarrow \mathbb{R}^{n}, g: J \times \mathcal{C} \rightarrow \mathbb{R}^{n}$ are given continuous functions, $\phi \in C(\tilde{J}), \varphi:[0, a] \rightarrow \mathbb{R}^{n}, \psi:[0, b] \rightarrow \mathbb{R}^{n}$ are given absolutely continuous functions with $\varphi(x)=\phi(x, 0), \psi(y)=\phi(0, y)$ for each $x \in[0, a], y \in[0, b]$, and $\mathcal{C}:=C([-\alpha, 0] \times[-\beta, 0])$ is the Banach space of continuous functions on $[-\alpha, 0] \times[-\beta, 0]$ coupled with the norm

$$
\|w\|_{\mathcal{C}}=\sup _{(x, y) \in[-\alpha, 0] \times[-\beta, 0]}\|w(x, y)\| .
$$

If $u \in C([-\alpha, a] \times[-\beta, b]) ; \alpha, \beta, a, b>0$, then for any $(x, y) \in J$, define $u_{(x, y)}$ by

$$
u_{(x, y)}(s, t)=u(x+s, y+t) ; \quad(s, t) \in[-\alpha, 0] \times[-\beta, 0],
$$

here $u_{(x, y)}(\cdot, \cdot)$ represents the history of the state from time $(x-\alpha, y-\beta)$ up to the present time $(x, y)$.

Next, we consider the system of fractional-order neutral integro-differential equations with infinite delay of the form

$$
\begin{aligned}
& { }^{c} D_{\theta}^{r}\left[u(x, y)-g\left(x, y, u_{(x, y)}\right)\right]=f\left(x, y, I_{\theta}^{r} u(x, y), u_{(x, y)}\right) ; \quad(x, y) \in J, \\
& u(x, y)=\phi(x, y), \quad \text { if }(x, y) \in \tilde{J}^{\prime}:=((-\infty, a] \times(-\infty, b]) \backslash((0, a] \times(0, b]), \\
& \begin{cases}u(x, 0)=\varphi(x) ; & x \in[0, a], \\
u(0, y)=\psi(y) ; & y \in[0, b], \\
\varphi(0)=\psi(0),\end{cases}
\end{aligned}
$$

where $J, \varphi, \psi$ are as in the problem (3)-(5) and $\phi \in C\left(\tilde{J}^{\prime}\right), f: J \times \mathbb{R}^{n} \times \mathcal{B} \rightarrow \mathbb{R}^{n}, g: J \times \mathcal{B} \rightarrow$ $\mathbb{R}^{n}$ are given continuous functions, and $\mathcal{B}$ is called a phase space that will be specified in Section 4.

During the last two decades, many authors have considered the questions of existence, uniqueness, estimates of solutions, and dependence with respect to initial conditions of the solutions of differential and integral equations of two and three variables (see [15-19] and the references therein).

It is clear that more complicated partial differential systems with deviated variables and partial differential integral systems can be obtained from (3) and (6) by a suitable definition of $f$ and $g$. Barbashin [20] considered a class of partial integro-differential equations which appear in mathematical modeling of many applied problems (see [21], Section 19). Recently Pachpatte [22,23] considered some classes of partial functional differential equations which occur in a natural way in the description of many physical phenomena. 
We present the existence results for our problems based on the nonlinear alternative of the Leray-Schauder theorem. The present results extend those considered with integer order derivative $[6,9,16,24,25]$ and those with fractional derivative $[11,12,26]$.

\section{Preliminaries}

In this section, we introduce notations, definitions, and preliminary facts which are used throughout this paper. By $C(J)$ we denote the Banach space of all continuous functions from $J$ into $\mathbb{R}^{n}$ with the norm

$$
\|w\|_{J}=\sup _{(x, y) \in J}\|w(x, y)\|,
$$

where $\|\cdot\|$ denotes the usual supremum norm on $\mathbb{R}^{n}$.

Also, $E:=C([-\alpha, a] \times[-\beta, b])$ is a Banach space with the norm

$$
\|w\|_{E}=\sup _{(x, y) \in[-\alpha, a] \times[-\beta, b]}\|w(x, y)\| .
$$

As usual, by $A C(J)$ we denote the space of absolutely continuous functions from $J$ into $\mathbb{R}^{n}$ and $L^{1}(J)$ is the space of Lebesgue-integrable functions $w: J \rightarrow \mathbb{R}^{n}$ with the norm

$$
\|w\|_{L^{1}}=\int_{0}^{a} \int_{0}^{b}\|w(x, y)\| d y d x
$$

Definition 2.1 ([27]) Let $r=\left(r_{1}, r_{2}\right) \in(0, \infty) \times(0, \infty), \theta=(0,0)$, and $u \in L^{1}(J)$. The leftsided mixed Riemann-Liouville integral of order $r$ of $u$ is defined by

$$
\left(I_{\theta}^{r} u\right)(x, y)=\frac{1}{\Gamma\left(r_{1}\right) \Gamma\left(r_{2}\right)} \int_{0}^{x} \int_{0}^{y}(x-s)^{r_{1}-1}(y-t)^{r_{2}-1} u(s, t) d t d s,
$$

where $\Gamma(\cdot)$ is the (Euler's) gamma function defined by $\Gamma(\xi)=\int_{0}^{\infty} t^{\xi-1} e^{-t} d t ; \xi>0$.

In particular,

$$
\left(I_{\theta}^{\theta} u\right)(x, y)=u(x, y), \quad\left(I_{\theta}^{\sigma} u\right)(x, y)=\int_{0}^{x} \int_{0}^{y} u(s, t) d t d s ; \quad \text { for almost all }(x, y) \in J,
$$

where $\sigma=(1,1)$.

Note that if $u \in L^{1}(J)$, then $I_{\theta}^{r} u$ exists for all $r_{1}, r_{2} \in(0, \infty)$. Moreover, $I_{\theta}^{r} u \in C(J)$ provided $u \in C(J)$, and

$$
\left(I_{\theta}^{r} u\right)(x, 0)=\left(I_{\theta}^{r} u\right)(0, y)=0 ; \quad x \in[0, a], y \in[0, b] .
$$

Example 2.2 Let $\lambda, \omega \in(-1, \infty)$ and $r=\left(r_{1}, r_{2}\right) \in(0, \infty) \times(0, \infty)$. Then

$$
I_{\theta}^{r} x^{\lambda} y^{\omega}=\frac{\Gamma(1+\lambda) \Gamma(1+\omega)}{\Gamma\left(1+\lambda+r_{1}\right) \Gamma\left(1+\omega+r_{2}\right)} x^{\lambda+r_{1}} y^{\omega+r_{2}} \quad \text { for almost all }(x, y) \in J .
$$

By $1-r$ we mean $\left(1-r_{1}, 1-r_{2}\right) \in[0,1) \times[0,1)$. 
Definition 2.3 ([27]) Let $r \in(0,1] \times(0,1]$ and $u \in L^{1}(J)$. The Caputo fractional-order derivative of order $r$ of $u$ is defined by the expression

$$
{ }^{c} D_{\theta}^{r} u(x, y)=\left(I_{\theta}^{1-r} D_{x y}^{2} u\right)(x, y)=\frac{1}{\Gamma\left(1-r_{1}\right) \Gamma\left(1-r_{2}\right)} \int_{0}^{x} \int_{0}^{y} \frac{D_{s t}^{2} u(s, t)}{(x-s)^{r_{1}}(y-t)^{r_{2}}} d t d s .
$$

The case $\sigma=(1,1)$ is included, and we have

$$
\left({ }^{c} D_{\theta}^{\sigma} u\right)(x, y)=\left(D_{x y}^{2} u\right)(x, y) \quad \text { for almost all }(x, y) \in J .
$$

Example 2.4 Let $\lambda, \omega \in(-1, \infty)$ and $r=\left(r_{1}, r_{2}\right) \in(0,1] \times(0,1]$. Then

$$
{ }^{c} D_{\theta}^{r} x^{\lambda} y^{\omega}=\frac{\Gamma(1+\lambda) \Gamma(1+\omega)}{\Gamma\left(1+\lambda-r_{1}\right) \Gamma\left(1+\omega-r_{2}\right)} x^{\lambda-r_{1}} y^{\omega-r_{2}} \quad \text { for almost all }(x, y) \in J .
$$

In the sequel, we need the following lemma.

Lemma 2.5 ([26]) Let $f \in L^{1}(J)$ and $g \in A C(J)$. Then the unique solution $u \in A C(J)$ of the problem

$$
\left\{\begin{array}{l}
{ }^{c} D_{\theta}^{r}(u-g)(x, y)=f(x, y) ; \quad(x, y) \in J, \\
u(x, 0)=\varphi(x) ; \quad x \in[0, a], \quad u(0, y)=\psi(y) ; \quad y \in[0, b], \\
\varphi(0)=\psi(0),
\end{array}\right.
$$

is given by the following expression:

$$
u(x, y)=\mu(x, y)+g(x, y)-g(x, 0)-g(0, y)+g(0,0)+\left(I_{\theta}^{r} f\right)(x, y) ; \quad(x, y) \in J,
$$

where

$$
\mu(x, y)=\varphi(x)+\psi(y)-\varphi(0) .
$$

As a consequence of Lemma 2.5, it is not difficult to verify the following result.

Corollary 2.6 Let $f \in L^{1}\left(J \times \mathbb{R}^{n} \times \mathcal{C}\right)$ and $g \in A C(J \times \mathcal{C})$. A function $u \in A C([-\alpha, a] \times$ $[-\beta, b])$ is a solution of the problem (3)-(5) if and only if $u$ satisfies

$$
\left\{\begin{aligned}
u(x, y)= & \Phi(x, y) ; \quad(x, y) \in \tilde{J}, \\
u(x, y)= & \mu(x, y)+g\left(x, y, u_{(x, y)}\right)+g\left(0,0, u_{(0,0)}\right) \\
& -g\left(x, 0, u_{(x, 0)}\right)-g\left(0, y, u_{(0, y)}\right) \\
& +I_{\theta}^{r} f\left(x, y, I_{\theta}^{r} u(x, y), u_{(x, y)}\right) ; \quad(x, y) \in J .
\end{aligned}\right.
$$

Also, we need the following theorem.

Theorem 2.7 (Nonlinear alternative of Leray-Schauder type [28]) By $\bar{U}$ and $\partial U$ we denote the closure of $U$ and the boundary of $U$ respectively. Let $X$ be a Banach space and $C$ a 
nonempty convex subset of $X$. Let $U$ be a nonempty open subset of $C$ with $0 \in U$ and $T$ : $\bar{U} \rightarrow C$ be a completely continuous operator.

Then either

(a) $T$ has fixed points or

(b) there exist $u \in \partial U$ and $\lambda \in(0,1)$ with $u=\lambda T(u)$.

\section{Existence results with finite delay}

Let us start by defining what we mean by a solution of the problem (3)-(5).

Definition 3.1 A function $u \in E$ is said to be a solution of the problem (3)-(5) if $u$ satisfies equations (3), (5) on $J$ and the condition (4) on $\tilde{J}$.

Further, we present conditions for the existence of a solution of the problem (3)-(5).

$\left(\mathrm{H}_{1}\right)$ There exist nonnegative functions $p, q, d \in C(J)$ such that

$$
\|f(x, y, u, v)\| \leq p(x, y)+q(x, y)\|u\|+d(x, y)\|v\|_{\mathcal{C}}
$$

for all $(x, y) \in J, u \in \mathbb{R}^{n}$, and $v \in \mathcal{C}$.

$\left(\mathrm{H}_{2}\right)$ For any bounded set $B$ in $E$, the set $\left\{(x, y) \rightarrow g\left(x, y, u_{(x, y)}\right): u \in B\right\}$ is equicontinuous in $E$, and there exist constants $L_{1}, L_{2} \geq 0$ such that

$$
\|g(x, y, u)\| \leq L_{1}+L_{2}\|u\|_{\mathcal{C}} ; \quad(x, y) \in J \text { and } u \in \mathcal{C} .
$$

Set

$$
L=\frac{a^{r_{1}} b^{r_{2}}}{\Gamma\left(1+r_{1}\right) \Gamma\left(1+r_{2}\right)}, \quad p^{*}=\|p\|_{J}, \quad q^{*}=\|q\|_{J} \quad \text { and } \quad d^{*}=\|d\|_{J} .
$$

Theorem 3.2 Assume that the hypotheses $\left(\mathrm{H}_{1}\right)$ and $\left(\mathrm{H}_{2}\right)$ hold. Then if

$$
4 L_{2}+q^{*} L^{2}+d^{*} L<1
$$

the problem (3)-(5) has at least one solution $u \in A C([-\alpha, a] \times[-\beta, b])$.

Proof Transform the problem (3)-(5) into a fixed point problem. Define the operator $N$ : $E \rightarrow E$ by

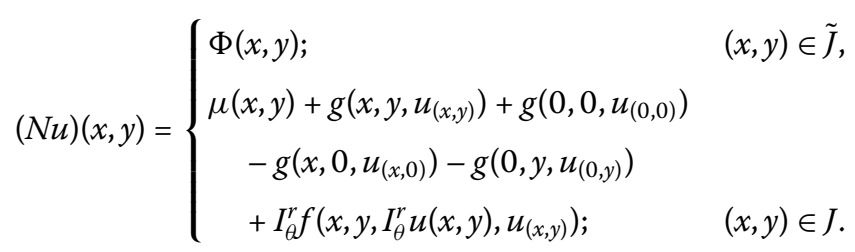

It is clear that $N$ maps $E$ into itself. By Corollary 2.6, the problem of finding the solutions of the problem (3)-(5) is reduced to finding the solutions of the operator equation $N(u)=u$. We shall show that the operator $N$ satisfies all the conditions of Theorem 2.7. The proof will be given in two steps. 
Step 1: $N$ is continuous and completely continuous.

Using $\left(\mathrm{H}_{2}\right)$ we deduce that $g$ is a complete continuous operator from $E$ to $\mathbb{R}^{n}$, so it suffices to show that the operator $N_{1}: E \rightarrow E$ defined by

$$
\left(N_{1} u\right)(x, y)= \begin{cases}\Phi(x, y) ; & (x, y) \in \tilde{J}, \\ \mu(x, y)+I_{\theta}^{r} f\left(x, y, I_{\theta}^{r} u(x, y), u_{(x, y)}\right) ; & (x, y) \in J\end{cases}
$$

is continuous and completely continuous. The proof will be given in several claims.

Claim 1: $N_{1}$ is continuous.

Let $\left\{u_{n}\right\}_{n \in \mathbb{N}}$ be a sequence such that $u_{n} \rightarrow u$ in $E$. Then for each $(x, y) \in[-\alpha, a] \times[-\beta, b]$, we have

$$
\begin{aligned}
& \|(\left.N_{1} u_{n}\right)(x, y)-\left(N_{1} u\right)(x, y) \| \\
& \leq\left\|I_{\theta}^{r}\left(f\left(x, y, I_{\theta}^{r} u_{n}(x, y), u_{n(x, y)}\right)-f\left(x, y, I_{\theta}^{r} u(x, y), u_{(x, y)}\right)\right)\right\| \\
& \leq \frac{1}{\Gamma\left(r_{1}\right) \Gamma\left(r_{2}\right)} \int_{0}^{x} \int_{0}^{y}(x-s)^{r_{1}-1}(y-t)^{r_{2}-1} \\
& \quad \times\left\|f\left(s, t, I_{\theta}^{r} u_{n}(s, t), u_{n(s, t)}\right)-f\left(s, t, I_{\theta}^{r} u(s, t), u_{(s, t)}\right)\right\| d t d s .
\end{aligned}
$$

Hence, for each $(x, y) \in J$, we get

$$
\begin{aligned}
\| N_{1}\left(u_{n}\right)- & N_{1}(u) \|_{E} \\
\leq & \frac{1}{\Gamma\left(r_{1}\right) \Gamma\left(r_{2}\right)} \int_{0}^{a} \int_{0}^{b}(x-s)^{r_{1}-1}(y-t)^{r_{2}-1} \\
& \times\left\|f\left(\cdot, \cdot, I_{\theta}^{r} u_{n}(\cdot, \cdot), u_{n(\cdot, \cdot)}\right)-f\left(\cdot, \cdot, I_{\theta}^{r} u(\cdot, \cdot), u_{(\cdot,)}\right)\right\|_{E} d t d s .
\end{aligned}
$$

Since $u_{n} \rightarrow u$ as $n \rightarrow \infty$ and $f, I_{\theta}^{r}$ are continuous, then

$$
\left\|N_{1}\left(u_{n}\right)-N_{1}(u)\right\|_{E} \rightarrow 0 \quad \text { as } n \rightarrow \infty
$$

Claim 2: $N_{1}$ maps bounded sets into bounded sets in $E$.

Indeed, it is enough to show that for any $\eta>0$, there exists a positive constant $\ell>0$ such that if $\|u\|_{E} \leq \eta$, we have that $\left\|N_{1}(u)\right\|_{E} \leq \ell$.

By $\left(\mathrm{H}_{2}\right)$ and $\left(\mathrm{H}_{3}\right)$, we have that for each $(x, y) \in J$ and $\|u\|_{E} \leq \eta$,

$$
\begin{aligned}
\left\|\left(N_{1} u\right)(x, y)\right\| \leq & \|\mu(x, y)\|+\frac{p^{*} a^{r_{1}} b^{r_{2}}}{\Gamma\left(1+r_{1}\right) \Gamma\left(1+r_{2}\right)} \\
& +\frac{q^{*} a^{r_{1}} b^{r_{2}}}{\Gamma\left(r_{1}\right) \Gamma\left(r_{2}\right) \Gamma\left(1+r_{1}\right) \Gamma\left(1+r_{2}\right)} \\
& \times \int_{0}^{x} \int_{0}^{y}(x-s)^{r_{1}-1}(y-t)^{r_{2}-1}\|u(s, t)\| d t d s \\
& +\frac{d^{*}}{\Gamma\left(r_{1}\right) \Gamma\left(r_{2}\right)} \int_{0}^{x} \int_{0}^{y}(x-s)^{r_{1}-1}(y-t)^{r_{2}-1}\left\|u_{(s, t)}\right\|_{\mathcal{C}} d t d s \\
\leq & \|\mu(x, y)\|+\frac{p^{*} a^{r_{1}} b^{r_{2}}}{\Gamma\left(1+r_{1}\right) \Gamma\left(1+r_{2}\right)}
\end{aligned}
$$




$$
\begin{aligned}
& \quad+\frac{q^{*} a^{r_{1}} b^{r_{2}}}{\Gamma\left(r_{1}\right) \Gamma\left(r_{2}\right) \Gamma\left(1+r_{1}\right) \Gamma\left(1+r_{2}\right)} \\
& \times \int_{0}^{x} \int_{0}^{y}(x-s)^{r_{1}-1}(y-t)^{r_{2}-1}\|u\|_{J} d t d s \\
& +\frac{d^{*}}{\Gamma\left(r_{1}\right) \Gamma\left(r_{2}\right)} \int_{0}^{x} \int_{0}^{y}(x-s)^{r_{1}-1}(y-t)^{r_{2}-1}\|u\|_{E} d t d s \\
& \leq\|\mu\|_{J}+p^{*} L+\left(q^{*} L^{2}+d^{*} L\right)\|u\|_{E} \\
& \leq\|\mu\|_{J}+p^{*} L+\left(q^{*} L+d^{*}\right) L \eta:=\ell_{1} .
\end{aligned}
$$

Thus,

$$
\left\|N_{1}(u)\right\|_{E} \leq \max \left\{\ell_{1},\|\Phi\|_{\mathcal{C}}\right\}:=\ell
$$

Claim 3: $N_{1}$ maps bounded sets in $E$ into equicontinuous sets in $E$.

Let $\left(x_{1}, y_{1}\right),\left(x_{2}, y_{2}\right) \in J, x_{1}<x_{2}, y_{1}<y_{2}, \eta>0$, and let $u \in E$ be such that $\|u\|_{E} \leq \eta$. Then

$$
\begin{aligned}
&\left\|\left(N_{1} u\right)\left(x_{2}, y_{2}\right)-\left(N_{1} u\right)\left(x_{1}, y_{1}\right)\right\| \\
& \leq \|\left\|\left(x_{2}, y_{2}\right)-\mu\left(x_{1}, y_{1}\right)\right\| \\
&+\frac{p^{*}+q^{*} L \eta+d^{*} \eta}{\Gamma\left(r_{1}\right) \Gamma\left(r_{2}\right)}\left[\int_{0}^{x_{1}} \int_{0}^{y_{1}}\left[\left(x_{2}-s\right)^{r_{1}-1}\left(y_{2}-t\right)^{r_{2}-1}-\left(x_{1}-s\right)^{r_{1}-1}\left(y_{1}-t\right)^{r_{2}-1}\right] d t d s\right. \\
&+\int_{x_{1}}^{x_{2}} \int_{y_{1}}^{y_{2}}\left(x_{2}-s\right)^{r_{1}-1}\left(y_{2}-t\right)^{r_{2}-1} d t d s+\int_{0}^{x_{1}} \int_{y_{1}}^{y_{2}}\left(x_{2}-s\right)^{r_{1}-1}\left(y_{2}-t\right)^{r_{2}-1} d t d s \\
&\left.+\int_{x_{1}}^{x_{2}} \int_{0}^{y_{1}}\left(x_{2}-s\right)^{r_{1}-1}\left(y_{2}-t\right)^{r_{2}-1} d t d s\right] \\
&=\left\|\mu\left(x_{1}, y_{1}\right)-\mu\left(x_{2}, y_{2}\right)\right\|+\frac{p^{*}+q^{*} L \eta+d^{*} \eta}{\Gamma\left(1+r_{1}\right) \Gamma\left(1+r_{2}\right)} \\
& \times\left[2 y_{2}^{r_{2}}\left(x_{2}-x_{1}\right)^{r_{1}}+2 x_{2}^{r_{1}}\left(y_{2}-y_{1}\right)^{r_{2}}+x_{1}^{r_{1}} y_{1}^{r_{2}}-x_{2}^{r_{1}} y_{2}^{r_{2}}-2\left(x_{2}-x_{1}\right)^{r_{1}}\left(y_{2}-y_{1}\right)^{r_{2}}\right] .
\end{aligned}
$$

As $x_{1} \rightarrow x_{2}, y_{1} \rightarrow y_{2}$, the right-hand side of the above inequality tends to zero with the same rate of convergence for all $u \in E$ with $\|u\|_{E} \leq \eta$.

The equicontinuity for the cases $x_{1}<x_{2}<0, y_{1}<y_{2}<0$ and $x_{1} \leq 0 \leq x_{2}, y_{1} \leq 0 \leq y_{2}$ is obvious. As a consequence of Claims 1 to 3 together with the Arzelá-Ascoli theorem, we can conclude that $N_{1}$ is continuous and completely continuous.

Step 2: A priori bounds.

We shall show that there exists an open set $U \subseteq E$ with $u \neq \lambda N(u)$ for all $\lambda \in(0,1)$ and all $u \in \partial U$.

Let $u \in E$ be such that $u=\lambda N(u)$ for some $0<\lambda<1$. Thus, for each $(x, y) \in J$, we have

$$
\begin{aligned}
u(x, y)= & \lambda\left(\mu(x, y)+g\left(x, y, u_{(x, y)}\right)+g\left(0,0, u_{(0,0)}\right)\right. \\
& \left.-g\left(x, 0, u_{(x, 0)}\right)-g\left(0, y, u_{(0, y)}\right)\right) \\
& +\lambda I_{\theta}^{r} f\left(x, y, I_{\theta}^{r} u(x, y), u_{(x, y)}\right) .
\end{aligned}
$$


Then for $(x, y) \in J$, we have

$$
\begin{aligned}
\|u(x, y)\| \leq & \|\mu(x, y)\|+4 L_{1}+L_{2}\left(\left\|u_{(x, y)}\right\|_{\mathcal{C}}+\left\|u_{(x, 0)}\right\|_{\mathcal{C}}+\left\|u_{(0, y)}\right\|_{\mathcal{C}}+\left\|u_{(0,0)}\right\|_{\mathcal{C}}\right) \\
& +p^{*} L+\frac{q^{*} L}{\Gamma\left(r_{1}\right) \Gamma\left(r_{2}\right)} \int_{0}^{x} \int_{0}^{y}(x-s)^{r_{1}-1}(y-t)^{r_{2}-1}\|u(s, t)\| d t d s \\
& +\frac{d^{*}}{\Gamma\left(r_{1}\right) \Gamma\left(r_{2}\right)} \int_{0}^{x} \int_{0}^{y}(x-s)^{r_{1}-1}(y-t)^{r_{2}-1}\left\|u_{(s, t)}\right\|_{\mathcal{C}} d t d s \\
\leq & \|\mu(x, y)\|+4 L_{1}+4 L_{2}\|u\|_{E}+p^{*} L \\
& +\frac{q^{*} L}{\Gamma\left(r_{1}\right) \Gamma\left(r_{2}\right)} \int_{0}^{x} \int_{0}^{y}(x-s)^{r_{1}-1}(y-t)^{r_{2}-1}\|u\|_{J} d t d s \\
& +\frac{d^{*}}{\Gamma\left(r_{1}\right) \Gamma\left(r_{2}\right)} \int_{0}^{x} \int_{0}^{y}(x-s)^{r_{1}-1}(y-t)^{r_{2}-1}\|u\|_{E} d t d s \\
\leq & \|\mu\|_{J}+4 L_{1}+p^{*} L+\left(4 L_{2}+q^{*} L^{2}+d^{*} L\right)\|u\|_{E} .
\end{aligned}
$$

It is obvious that

$$
\|u\|_{E}=\max \left\{\|u\|_{\tilde{I}},\|u\|_{J}\right\} \leq \max \left\{\|\phi\|_{\tilde{J}},\|u\|_{J}\right\}
$$

As consequence, if $\|u\|_{J} \leq\|\phi\|_{\tilde{j}}$, then $\|u\|_{E} \leq\|\phi\|_{\tilde{j}}$.

On the contrary, when $\|u\|_{J}>\|\phi\|_{\tilde{j}}$, we have that $\|u\|_{E}=\|u\|_{J}$. So, from the previous inequalities and the condition (9), we arrive at

$$
\|u\|_{E} \leq \frac{\|\mu\|_{J}+4 L_{1}+p^{*} L}{1-4 L_{2}-q^{*} L^{2}-d^{*} L}:=M .
$$

Thus,

$$
\|u\|_{E} \leq \max \left\{\|\phi\|_{\tilde{j}}, M\right\}:=M^{*}
$$

Set

$$
U=\left\{u \in E:\|u\|_{E}<M^{*}+1\right\} .
$$

By our choice of $U$, there is no $u \in \partial U$ such that $u=\lambda N(u)$ for $\lambda \in(0,1)$.

As a consequence of Steps 1 and 2 together with Theorem 2.7, we deduce that $N$ has a fixed point $u$ in $\bar{U}$ which is a solution to the problem (3)-(5).

\section{The phase space $\mathcal{B}$}

The notation of the phase space $\mathcal{B}$ plays an important role in the study of both qualitative and quantitative theory for functional differential equations. A usual choice is a seminormed space satisfying suitable axioms, which was introduced by Hale and Kato (see [7]). For further applications, see, for instance, the books $[10,29,30]$ and their references. For any $(x, y) \in J$, denote $\mathcal{E}_{(x, y)}:=([0, x] \times\{0\}) \cup(\{0\} \times[0, y])$. Furthermore, in case $x=a$, $y=b$, we write simply $\mathcal{E}$. Consider the space $\left(\mathcal{B},\|(\cdot, \cdot)\|_{\mathcal{B}}\right)$ a semi-normed linear space of functions mapping $(-\infty, 0] \times(-\infty, 0]$ into $\mathbb{R}^{n}$ and satisfying the following fundamental axioms which were adapted from those introduced by Hale and Kato for ordinary differential functional equations: 
$\left(\mathrm{A}_{1}\right)$ If $z:(-\infty, a] \times(-\infty, b] \rightarrow \mathbb{R}^{n}$ is a continuous function on $J$ and $z_{(x, y)} \in \mathcal{B}$ for all $(x, y) \in \mathcal{E}$, then there are constants $H, K, M>0$ such that for any $(x, y) \in J$, the following conditions hold:

(i) $z_{(x, y)}$ is in $\mathcal{B}$;

(ii) $\|z(x, y)\| \leq H\left\|z_{(x, y)}\right\|_{\mathcal{B}}$;

(iii) $\left\|z_{(x, y)}\right\|_{\mathcal{B}} \leq K \sup _{(s, t) \in[0, x] \times[0, y]}\|z(s, t)\|+M \sup _{(s, t) \in \mathcal{E}_{(x, y)}}\left\|z_{(s, t)}\right\|_{\mathcal{B}}$

$\left(\mathrm{A}_{2}\right)$ For the function $z(\cdot, \cdot)$ in $\left(\mathrm{A}_{1}\right), z_{(x, y)}$ is a $\mathcal{B}$-valued continuous function on $J$.

$\left(\mathrm{A}_{3}\right)$ The space $\mathcal{B}$ is complete.

Now, we present some examples of phase spaces $[6,9]$.

Example 4.1 Let $\mathcal{B}$ be the set of all functions $\phi:(-\infty, 0] \times(-\infty, 0] \rightarrow \mathbb{R}^{n}$ which are continuous on $[-\alpha, 0] \times[-\beta, 0], \alpha, \beta \geq 0$, with the semi-norm

$$
\|\phi\|_{\mathcal{B}}=\sup _{(s, t) \in[-\alpha, 0] \times[-\beta, 0]}\|\phi(s, t)\| .
$$

Then we have $H=K=M=1$. The quotient space $\widehat{\mathcal{B}}=\mathcal{B} /\|\cdot\|_{\mathcal{B}}$ is isometric to the space $\mathcal{C}$ of all continuous functions from $[-\alpha, 0] \times[-\beta, 0]$ into $\mathbb{R}^{n}$ with the supremum norm. This means that partial differential functional equations with finite delay are included in our axiomatic model.

Example 4.2 Let $\gamma \in \mathbb{R}$, and let $C_{\gamma}$ be the set of all continuous functions $\phi:(-\infty, 0] \times$

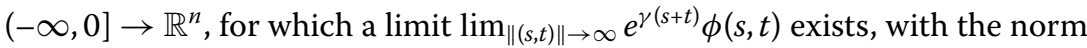

$$
\|\phi\|_{C_{\gamma}}=\sup _{(s, t) \in(-\infty, 0] \times(-\infty, 0]} e^{\gamma(s+t)}\|\phi(s, t)\| .
$$

Then we have $H=1$ and $K=M=\max \left\{e^{-\gamma(a+b)}, 1\right\}$.

Example 4.3 Let $\alpha, \beta, \gamma \geq 0$, and let

$$
\|\phi\|_{C L_{\gamma}}=\sup _{(s, t) \in[-\alpha, 0] \times[-\beta, 0]}\|\phi(s, t)\|+\int_{-\infty}^{0} \int_{-\infty}^{0} e^{\gamma(s+t)}\|\phi(s, t)\| d t d s
$$

be the semi-norm for the space $C L_{\gamma}$ of all functions $\phi:(-\infty, 0] \times(-\infty, 0] \rightarrow \mathbb{R}^{n}$ which are continuous on $[-\alpha, 0] \times[-\beta, 0]$ measurable on $((-\infty,-\alpha] \times(-\infty, 0]) \cup((-\infty, 0] \times$ $(-\infty,-\beta])$, and such that $\|\phi\|_{C L_{\gamma}}<\infty$. Then

$$
H=1, \quad K=\int_{-\alpha}^{0} \int_{-\beta}^{0} e^{\gamma(s+t)} d t d s, \quad M=2 .
$$

\section{Existence results with infinite delay}

Set

$$
\Omega:=\left\{u:(-\infty, a] \times(-\infty, b] \rightarrow \mathbb{R}^{n}: u_{(x, y)} \in \mathcal{B} \text { for }(x, y) \in \mathcal{E} \text { and } u_{\left.\right|_{J}} \in C(J)\right\}
$$

Let us start by defining what we mean by a solution of the problem (6)-(8). 
Definition 5.1 A function $u \in \Omega$ is said to be a solution of (6)-(8) if $u$ satisfies equations (6) and (8) on $J$ and the condition (7) on $\tilde{J}^{\prime}$.

Now, we present conditions for the existence of a solution of the problem (6)-(8).

$\left(\mathrm{H}_{1}^{\prime}\right)$ There exist nonnegative functions $\bar{p}, \bar{q}, \bar{d} \in C(J)$ such that

$$
\|f(x, y, u, v)\| \leq \bar{p}(x, y)+\bar{q}(x, y)\|u\|+\bar{d}(x, y)\|v\|_{\mathcal{B}}
$$

for all $(x, y) \in J, u \in \mathbb{R}^{n}$, and $v \in \mathcal{B}$.

$\left(\mathrm{H}_{2}^{\prime}\right)$ For any bounded set $B$ in $\Omega$, the set $\left\{(x, y) \rightarrow g\left(x, y, u_{(x, y)}\right): u \in B\right\}$ is equicontinuous in $\Omega$, and there exist constants $\bar{L}_{1}, \bar{L}_{2} \geq 0$ such that

$$
\|g(x, y, u)\| \leq \bar{L}_{1}+\bar{L}_{2}\|u\|_{\mathcal{B}} ; \quad(x, y) \in J \text { and } u \in \mathcal{B} .
$$

Set

$$
\bar{p}^{*}=\|\bar{p}\|_{J}, \quad \bar{q}^{*}=\|\bar{q}\|_{J} \quad \text { and } \quad \bar{d}^{*}=\|\bar{d}\|_{J} .
$$

Theorem 5.2 Assume that the hypotheses $\left(\mathrm{H}_{1}^{\prime}\right)$ and $\left(\mathrm{H}_{2}^{\prime}\right)$ hold. If

$$
4 \bar{L}_{2}+\bar{q}^{*} L^{2}+\bar{d}^{*} L<1
$$

then the problem (6)-(8) has at least one solution on $(-\infty, a] \times(-\infty, b]$.

Proof Transform the problem (6)-(8) into a fixed point problem. Let $u \in \Omega$ and define the operator $\bar{N}: \Omega \rightarrow \Omega$ by

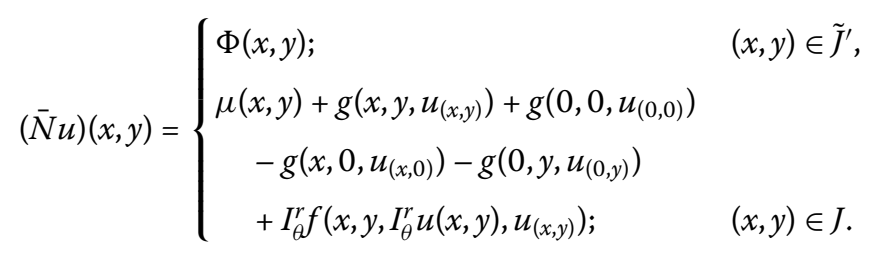

As in Theorem 3.2, we can easily see that $\bar{N}$ maps $\Omega$ into itself.

Let $v(\cdot, \cdot):(-\infty, a] \times(-\infty, b] \rightarrow \mathbb{R}^{n}$ be a function defined by

$$
v(x, y)= \begin{cases}\phi(x, y), & (x, y) \in \tilde{J}^{\prime}, \\ \mu(x, y), & (x, y) \in J .\end{cases}
$$

Then $v_{(x, y)}=\phi$ for all $(x, y) \in \mathcal{E}$.

For each $w \in C(J)$ with $w(x, y)=0$ for each $(x, y) \in \mathcal{E}$, we denote by $\bar{w}$ the function defined by

$$
\bar{w}(x, y)= \begin{cases}0, & (x, y) \in \tilde{J}^{\prime}, \\ w(x, y), & (x, y) \in J .\end{cases}
$$

If $u(\cdot, \cdot)$ satisfies the integral equation, $u(x, y)=(\bar{N} u)(x, y) ;(x, y) \in[-\alpha, a] \times[-\beta, b]$, we can 
decompose $u(\cdot, \cdot)$ as $u(x, y)=\bar{w}(x, y)+v(x, y) ;(x, y) \in[-\alpha, a] \times[-\beta, b]$, which implies $u_{(x, y)}=$ $\bar{w}_{(x, y)}+v_{(x, y)}$ for every $(x, y) \in J$, and the function $w(\cdot, \cdot)$ satisfies

$$
\begin{aligned}
w(x, y)= & g\left(x, y, u_{(x, y)}\right)+g\left(0,0, \bar{w}_{(0,0)}+v_{(0,0)}\right) \\
& -g\left(x, 0, \bar{w}_{(x, 0)}+v_{(x, 0)}\right)-g\left(0, y, \bar{w}_{(0, y)}+v_{(0, y)}\right) \\
& +I_{\theta}^{r} f\left(x, y, I_{\theta}^{r}(\bar{w}(x, y)+v(x, y)), \bar{w}_{(x, y)}+v_{(x, y)}\right) .
\end{aligned}
$$

Set

$$
C_{0}=\{w \in C(J): w(x, y)=0 \text { for }(x, y) \in \mathcal{E}\},
$$

and let $\|\cdot\|_{(a, b)}$ be the norm in $C_{0}$ defined by

$$
\|w\|_{(a, b)}=\sup _{(x, y) \in \mathcal{E}}\left\|w_{(x, y)}\right\|_{\mathcal{B}}+\sup _{(x, y) \in J}\|w(x, y)\|=\sup _{(x, y) \in J}\|w(x, y)\|, \quad w \in C_{0} .
$$

$C_{0}$ is a Banach space with the norm $\|\cdot\|_{(a, b)}$.

Note that $u \in \Omega$ if and only if $w \in C_{0}$.

Let the operator $P: C_{0} \rightarrow C_{0}$ be defined by

$$
\begin{aligned}
(P w)(x, y)= & g\left(x, y, u_{(x, y)}\right)+g\left(0,0, \bar{w}_{(0,0)}+v_{(0,0)}\right) \\
& -g\left(x, 0, \bar{w}_{(x, 0)}+v_{(x, 0)}\right)-g\left(0, y, \bar{w}_{(0, y)}+v_{(0, y)}\right) \\
& +\frac{1}{\Gamma\left(r_{1}\right) \Gamma\left(r_{2}\right)} \int_{0}^{x} \int_{0}^{y}(x-s)^{r_{1}-1}(y-t)^{r_{2}-1} \\
& \times f\left(s, t, I_{\theta}^{r}(\bar{w}(s, t)+v(s, t)), \bar{w}_{(s, t)}+v_{(s, t)}, g(s, t)\right) d t d s .
\end{aligned}
$$

Then the operator $\bar{N}$ has a fixed point in $\Omega$ if and only if $P$ has a fixed point in $C_{0}$. As in the proof of Theorem 3.2, we can show that the operator $P$ satisfies all the conditions of Theorem 2.7. Indeed, to prove that $P$ is continuous and completely continuous and by using $\left(\mathrm{H}_{2}^{\prime}\right)$, it suffices to show that the operator $\bar{P}: F \rightarrow F$ defined by

$$
\begin{aligned}
(\bar{P} w)(x, y)= & \frac{1}{\Gamma\left(r_{1}\right) \Gamma\left(r_{2}\right)} \int_{0}^{x} \int_{0}^{y}(x-s)^{r_{1}-1}(y-t)^{r_{2}-1} \\
& \times f\left(s, t, I_{\theta}^{r}(\bar{w}(s, t)+v(s, t)), \bar{w}_{(s, t)}+v_{(s, t)}, g(s, t)\right) d t d s
\end{aligned}
$$

is continuous and completely continuous. Also, we can show that there exists an open set $U^{\prime} \subseteq F$ with $u \neq \lambda P(u)$ for $\lambda \in(0,1)$ and $u \in \partial U^{\prime}$. Consequently, by Theorem 2.7, we deduce that $\bar{N}$ has a fixed point $u$ in $\overline{U^{\prime}}$ which is a solution to the problem (6)-(8).

\section{An example}

Consider the following neutral integro-differential equations of fractional order:

$$
\begin{aligned}
& { }^{c} D_{\theta}^{r}\left(u(x, y)-\frac{1+2|u(x-1, y-2)|}{4 e^{x+y+10}}\right) \\
& \quad=\frac{3+(x+y)|u(x-1, y-2)|+x y||_{\theta}^{r} u(x, y) \mid}{e^{10}\left(1+|u(x-1, y-2)|+\left|I_{\theta}^{r} u(x, y)\right|\right)} ; \quad(x, y) \in[0,1] \times[0,1],
\end{aligned}
$$




$$
\begin{aligned}
& u(x, y)=x+y^{2} ; \quad \text { if }(x, y) \in \tilde{J}:=([-1,1] \times[-2,1]) \backslash((0,1] \times(0,1]), \\
& \varphi(x)=x, \quad \psi(y)=y^{2} ; \quad x, y \in[0,1] .
\end{aligned}
$$

Set

$$
f(x, y, u, v)=\frac{3+(x+y)|u|+x y|v|}{e^{10}(1+|u|+|v|)} ; \quad(x, y) \in[0,1] \times[0,1],
$$

and

$$
g(x, y, u)=\frac{1+2|u|}{4 e^{x+y+10}} ; \quad(x, y) \in[0,1] \times[0,1] .
$$

We have $\mu(x, y)=x+y^{2} ;(x, y) \in[0,1] \times[0,1]$. For each $u, v \in \mathbb{R}$ and $(x, y) \in[0,1] \times[0,1]$, we have

$$
|f(x, y, u, v)| \leq e^{-10}(3+(x+y)|u|+x y|v|),
$$

and

$$
|g(x, y, u)| \leq \frac{1}{4} e^{-10}(1+2|u|)
$$

Hence, the condition $\left(\mathrm{H}_{1}\right)$ is satisfied with $p^{*}=3 e^{-10}, q^{*}=2 e^{-10}, d^{*}=e^{-10}$. Also, the condition $\left(\mathrm{H}_{2}\right)$ is satisfied with $L_{1}=\frac{1}{4} e^{-10}$ and $L_{2}=\frac{1}{2} e^{-10}$.

We shall show that the condition (9) holds for each $\left(r_{1}, r_{2}\right) \in(0,1] \times(0,1]$ with $a=b=1$. Indeed, $\Gamma\left(r_{i}\right)>0.7, \Gamma\left(1+r_{i}\right)>0.7 ; i=1,2$, and $L=\frac{1}{\Gamma\left(1+r_{1}\right) \Gamma\left(1+r_{2}\right)}<2.1$. Then

$$
4 L_{2}+q^{*} L^{2}+d^{*} L=2 e^{-10}+e^{-10}\left(2 L^{2}+L\right)<\frac{12.92}{e^{10}}<1
$$

Consequently, Theorem 3.2 implies that the problem (16)-(18) has at least one solution defined on $[-1,1] \times[-2,1]$.

\section{Competing interests}

The authors declare that they have no competing interests.

\section{Authors' contributions}

The three authors have participated into the obtained results. The collaboration of each one cannot be separated in different parts of the paper. All of them have made substantial contributions to the theoretical results. The three authors have been involved in drafting the manuscript and revising it critically for important intellectual content. All authors have given final approval of the version to be published.

\section{Author details}

'Laboratoire de Mathématiques, Université de Saïda, B.P. 138, Saïda, 20000, Algeria. ${ }^{2}$ Laboratoire de Mathématiques, Université de Sidi Bel-Abbès, B.P. 89, Sidi Bel-Abbès, 22000, Algeria. ${ }^{3}$ Departamento de Análise Matemática, Facultade de Matemáticas, Universidade de Santiago de Compostela, Santiago de Compostela, Spain.

\section{Acknowledgements}

The authors are grateful to the referees for their helpful remarks. Third author is partially supported by FEDER and Ministerio de Educación y Ciencia, Spain, project MTM2010-15314. 


\section{References}

1. Abbas, S, Benchohra, M, N'Guérékata, GM: Topics in Fractional Differential Equations. Springer, New York (2012)

2. Baleanu, D, Diethelm, K, Scalas, E, Trujillo, JJ: Fractional Calculus Models and Numerical Methods. World Scientific, New York (2012)

3. Kilbas, AA, Srivastava, HM, Trujillo, JJ: Theory and Applications of Fractional Differential Equations. Elsevier, Amsterdam (2006)

4. Lakshmikantham, V, Leela, S, Vasundhara, J: Theory of Fractional Dynamic Systems. Cambridge Academic Publishers, Cambridge (2009)

5. Podlubny, I: Fractional Differential Equations. Academic Press, San Diego (1999)

6. Czlapinski, T: On the Darboux problem for partial differential-functional equations with infinite delay at derivatives. Nonlinear Anal. 44, 389-398 (2001)

7. Hale, J, Kato, J: Phase space for retarded equations with infinite delay. Funkc. Ekvacioj 21, 11-41 (1978)

8. Corduneau, C, Lakshmikantham, V: Equations with unbounded delay: survey. Nonlinear Anal. 4, 831-877 (1980)

9. Czlapinski, T: Existence of solutions of the Darboux problem for partial differential-functional equations with infinite delay in a Banach space. Comment. Math. Prace Mat. 35, 111-122 (1995)

10. Hino, Y, Murakami, S, Naito, T: Functional Differential Equations with Infinite Delay. Lecture Notes in Mathematics, vol. 1473. Springer, Berlin (1991)

11. Abbas, S, Benchohra, M: Partial hyperbolic differential equations with finite delay involving the Caputo fractional derivative. Commun. Math. Anal. 7, 62-72 (2009)

12. Abbas, S, Benchohra, M: Darboux problem for perturbed partial differential equations of fractional order with finite delay. Nonlinear Anal. Hybrid Syst. 3, 597-604 (2009)

13. Abbas, S, Agarwal, RP, Benchohra, M: Darboux problem for impulsive partial hyperbolic differential equations of fractional order with variable times and infinite delay. Nonlinear Anal. Hybrid Syst. 4, 818-829 (2010)

14. Abbas, S, Benchohra, M: Darboux problem for partial functional differential equations with infinite delay and Caputo's fractional derivative. Adv. Dyn. Syst. Appl. 5(1), 1-19 (2010)

15. Beesack, PR: Systems of multidimensional Volterra integral equations and inequalities. Nonlinear Anal. 9, 1451-1486 (1985)

16. Kamont, Z: Hyperbolic Functional Differential Inequalities and Applications. Kluwer Academic, Dordrecht (1999)

17. Kwapisz, M: Weighted norms and existence and uniqueness of $L^{p}$ solutions for integral equations in several variables. J. Differ. Equ. 97, 246-262 (1992)

18. Pachpatte, BG: Inequalities for Differential and Integral Equations. Academic Press, New York (1998)

19. Pachpatte, BG: Integral and Finite Difference Inequalities and Applications. North-Holland Mathematics Studies, vol. 205. Elsevier, Amsterdam (2006)

20. Barbashin, EA: On conditions for the conservation of stability of solutions to integrodifferential equations. Izv. VUZov. Mat. 1, 25-34 (1957) (in Russian)

21. Appell, JM, Kalitvin, AS, Zabrejko, PP: Partial Integral Operators and Integrodifferential Equations. Dekker, New York (2000)

22. Pachpatte, BG: On a general partial integral equation of Barbashin type. Tamsui Oxf. J. Inf. Math. Sci. 27(1), 99-115 (2011)

23. Pachpatte, BG: On a certain hyperbolic partial differential equation. Tamsui Oxf. J. Inf. Math. Sci. 25(1), 39-54 (2009)

24. Kamont, Z, Kropielnicka, K: Differential difference inequalities related to hyperbolic functional differential systems and applications. Math. Inequal. Appl. 8(4), 655-674 (2005)

25. Lakshmikantham, V, Pandit, SG: The method of upper, lower solutions and hyperbolic partial differential equations. J. Math. Anal. Appl. 105, 466-477 (1985)

26. Abbas, S, Benchohra, M, Zhou, Y: Darboux problem for fractional order neutral functional partial hyperbolic differential equations. Int. J. Dyn. Syst. Differ. Equ. 2(3-4), 301-312 (2009)

27. Vityuk, AN, Golushkov, AV: Existence of solutions of systems of partial differential equations of fractional order Nonlinear Oscil. 7, 318-325 (2004)

28. Granas, A, Dugundji, J: Fixed Point Theory. Springer, New York (2003)

29. Hale, JK, Verduyn Lunel, S: Introduction to Functional Differential Equations. Applied Mathematical Sciences, vol. 99. Springer, New York (1993)

30. Lakshmikantham, V, Wen, L, Zhang, B: Theory of Differential Equations with Unbounded Delay. Mathematics and Its Applications. Kluwer Academic, Dordrecht (1994)

doi:10.1186/1687-2770-2012-128

Cite this article as: Abbas et al.: Partial neutral functional integro-differential equations of fractional order with

delay. Boundary Value Problems 2012 2012:128. 